\title{
Tumor-Associated Macrophages, Inflammation and Pathogenesis of Hepatocellular Carcinoma
}

\author{
Matthieu Lewis $^{1,2}$ and Aksam J Merched ${ }^{1,2,3^{*}}$ \\ ${ }^{1}$ INSERM Liver Research Group, U1053, Bordeaux, France \\ ${ }^{2}$ Liver Research Group, Unités Mixtes de Recherche 1053, Bordeaux University, Bordeaux, France \\ ${ }^{3}$ Department of Molecular and Cellular Biology, Baylor College of Medicine, Houston, TX, USA
}

*Corresponding author: Aksam J Merched, Department of Pharmaceutical Sciences, Bordeaux University and INSERM1053 (Liver Research Group), 146 rue Léo Saignat, 33076 Bordeaux cedex, France, Tel: +33 (0)557571771; Fax: +33 (0)556614077; E-mail: aksam.merched@inserm.fr

Received date: August 04, 2014; Accepted date: October 06, 2014; Published date: October 15, 2014

Copyright: ( 2014 Lewis M, et al. This is an open-access article distributed under the terms of the Creative Commons Attribution License, which permits unrestricted use, distribution, and reproduction in any medium, provided the original author and source are credited.

\begin{abstract}
Chronic inflammatory state is linked to the emergence of hepatocellular carcinoma, one of the most common and aggressive cancers worldwide. The complex microenvironment of tumor changes dynamically and consequently affects the pathological process. Understanding the immunological milieu of tumor in hepatocellular carcinoma can have crucial repercussions on how we see liver cancer and help plan for future cancer treatments. Taking part in this dynamic microenvironment, macrophages play a vital role in tumor growth and proliferation, cancer survival, metastasis and angiogenesis. In this review, we discuss the place and the current understanding of tumorassociated macrophages and their role in the process of hepatocarcinogenesis. In addition, we present directions for research targeting macrophage plasticity for future anti-tumor therapeutic approaches.
\end{abstract}

Keywords: Hepatocellular carcinoma; Carcinogenesis; Macrophage polarization; Innate immunity; Inflammation

\section{Introduction}

Hepatocellular carcinoma (HCC) is one of the most aggressive cancers worldwide. It is held responsible for up to 1 million deaths globally per annum [1]. For men, it is the fifth most frequently diagnosed cancer worldwide and the second most deadly, whereas for adult women it is the seventh most frequently diagnosed cancer and only the sixth most fatal [2]. HCC occurs mainly after hepatic stress or injury that induce fibrosis and/or cirrhosis. Stresses on the liver can come from many different sources; the virus hepatitis B [3] and hepatitis C [4] are major causes of HCC development. Also, chronic alcohol consumption can worsen the outcome dramatically [5] by causing liver cirrhosis. Additional diseases are linked to HCC development, like biliary diseases including primary biliary cirrhosis [6], metabolic disorders like obesity [7] and genetic conditions such as hereditary hemochromatosis [8].

HCC is an inflammation-related cancer, as a chronic inflammatory state is necessary for cancer appearance. This review highlights the place of inflammation in hepatocellular carcinoma. We inspect the molecular cross talk in the tumor microenvironment between the hepatocellular carcinoma and the immunological cells, mainly tumorassociation macrophages and it implication in hepatocarcinogenesis.

\section{Emergence of inflammatory responses as a new hallmark of cancer}

For many years, scientists have tried to understand the pathological characteristics of cancer. Determining and understanding specific events that take place in order for a tumor to arise and survive is fundamental. Certain crucial events have been identified to be essential in order for the cancer to grow. These events have been portrayed as the "hallmarks of cancer" and in the year 2000, Douglas Hanahan and Robert A Weinburg produced a list of six separate biological manifestations that lead to cancer growth [9]. Every cancer must, according to the two researchers, resist cell death, sustain proliferative signaling, evade growth suppressors, activate invasion and metastasis, enable replicative immortality and finally induce angiogenesis [9].

More recent studies start to shed light on additionally hallmarks of cancer and more particularly, the role of inflammation in cancer and the tumor's ability to bypass immune suppression. This suggestion was taken into account after the study of different cancer types in the 1980s. The pathologists found high levels of immune cells coming from both the innate and adaptive branches [10]. This lead to the hypothesis that tumor masses induce types of inflammatory reactions and that studying the immune cells in and around the tumor mass could help understand the specific role and function of this event. Thanks to more recent cell marking techniques and advances in research, scientists determined that there is a large range of various immune responses in different types of cancer [11]. These different types of responses vary in immune cell type and density [12], but can in most cases be used as a prognostic value generally determining poor outcome.

Upon studying these immune responses, scientists were intrigued by the paradoxical inflammatory response which increases tumor progression instead of controlling cancer cell proliferation [13]. Indeed, the inflammatory cells secrete factors that sustain cancer growth in many ways. They secrete cytotoxic reactive oxygen species (ROS) that increase mutational frequency in cancer cells, thus augmenting cancerous outcome [14], growth factors, angiogenesis boosting factors, anti-apoptotic factors and metastatic factors [15].

Not only do cancer cells program the surrounding inflammatory cells to release factors that promote and sustain tumor growth, they 
also evade eradication by the immune system. This hypothesis originated after studies found that immuno-compromised patients were also often diagnosed with cancer [16]. These observations elucidated that the immune system can also eradicate cancer formation; in fact, most cancers are destroyed by the immune system by a tissue protective response and immune surveillance [14]. The subsequent hypothesis was that cancer proliferation occurs generally after "immunoediting" which decreases the immunogenic capabilities of a patient. Studies on mice proved that cancers could alter immune surveillance in order to proliferate [17].

As an example of an inflammation-related cancer, HCC alter their surrounding immunological territory in order to accumulate and prosper. The growth and survival of these tumor cells is closely correlated with microenvironmental alterations in and around the tumor mass.

\section{Tumor microenvironment and tumor-associated macrophages in hepatocellular carcinoma}

Tumor cells in HCC are in a cross talk with their surrounding microenvironment, of complex cellular and chemical composition, which helps nurture neoplastic maintenance and survival. Thus, the HCC microenvironment is deeply involved in modulating all the processes of hepatocarcinogenesis. In and around tumor cells we can find growth factors and cytokines, extracellular matrix proteins, proteinases and a multitude of different cells [18]. Among the cells present are stromal cells, carcinoma-associated fibroblasts, hepatic stellate cells endothelial cells and, more importantly for our review, immune cells [18]. The immune cells consist of regulatory $(\mathrm{CD} 4+\mathrm{CD} 25+)$ and cytotoxic $\mathrm{T}(\mathrm{CD} 8+)$ cells, Kupffer cells and tumorassociated macrophages (TAMs). All these partners generate immune responses involved in the progression of HCC. Regulatory T cells, Kupffer cells (resident liver macrophages) and other TAMs impair cytotoxic $\mathrm{T}$ cell activities and their anticancer functions. Accordingly, several studies reported associations of decreased intratumoral densities of NK cells, low cytotoxic T, high TAM density and high regulatory $\mathrm{T}$ cell numbers with better pathological features and worse prognosis in HCC patients [19-22]. Recent studies have proven the importance of TAMs in HCC proliferation and survival $[14,15,18,21,23,24]$. TAMs belong to the macrophage lineage that are found in close proximity or within certain tumor masses [25]. They are generated from circulating monocytes in the bloodstream, precursors of macrophage differentiation, and are recruited to the tumor mass thanks to specific tumor-derived signals. Clinical studies have shown that the presence of large quantities of TAMs has been correlated with poor prognosis $[23,24,26]$. We are gaining more insights about the functions of TAMs; they can possess either procancer or anti-cancer roles, depending on a wide range of molecular growth factors, cytokines and chemokines, expressed in the tumor microenvironment [27]. They reply to these stimuli by undergoing two separate differentiation programs. Commonly, the terms used to define the two opposing profiles are Classically Activated Macrophages (CAMs) or M1 macrophages, and Alternatively Activated Macrophages (AAMs) or M2 macrophages [28]. These two macrophages represent the extremes of a continuum of macrophage activation, as numerous sub-types exist [29]. The two phenotypes differ based on their gene-expression profiles and cellular functions [30]. Both of these macrophage sub-types induce $\mathrm{T}$ helper immune responses, M1 macrophages induce $\mathrm{T}$ helper 1 cytotoxic immune response through the expression of many pro-inflammatory signals, whereas M2 macrophages trigger a T helper 2 immune response and secrete a number of anti-inflammatory signals, thus creating a procancer microenvironment by increasing angiogenesis, tissue remodeling and tissue repair (Figure 1) [31,32].

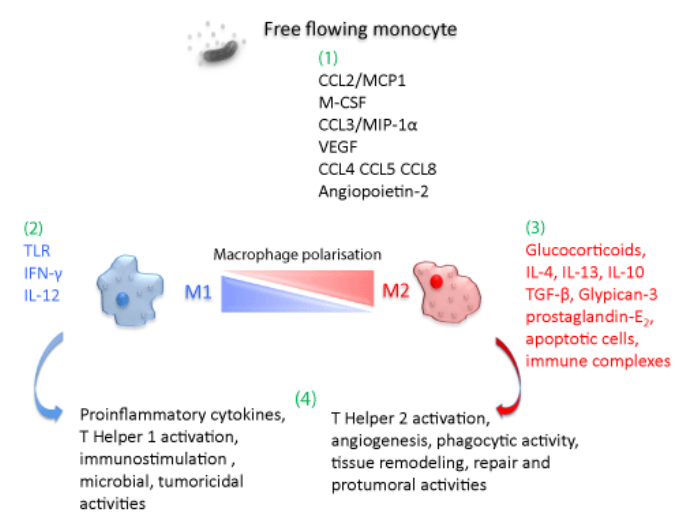

Figure 1: Monocyte recruitment, differentiation into macrophages and their functional involvement. The figure summarizes: (1) Secreted factors that recruit monocytes to tumor microenvironment, (2) Factors that increase M1 macrophage polarization, (3) Factors that increase M2 macrophage polarization and (4) specific functions of each type of macrophages.

Studies show that in and around the HCC microenvironment, TAMs are mainly polarized towards the M2 macrophage phenotype that helps promote tumor growth and survival [24]. Understanding which factors polarize the tumor-associated macrophages in one or the other direction is critical in order to better understand the molecular interactions that class these immune cells into the M1 cytotoxic or M2 pro-tumor sub-types.

\section{Tumor-associated macrophage recruitment, polarization and functions}

Most tumors are eradicated through a physiological cytotoxic T cell immune response which is promoted by tumor-associated macrophages. Unfortunately, some tumors evade this natural immune destruction by changing the tumor-associated macrophage phenotype in order to help promote tumor proliferation. The study of this phenomenon is crucial for understanding, first and foremost, which factors polarize tumor-associated macrophages to help maintain and promote tumor growth. Tumor-associated macrophages derive from free flowing monocytes in the blood and can be found in and around tumor masses [25]. They are the main type of inflammatory cells in tumor masses and play an essential role in tumor progression [24]. TAMs are recruited to the tumor by multiple signaling molecules. Among these molecules we can find Chemokine (C-C motif) Ligand 2 (CCL2)/Monocyte Chemotactic Protein 1 (MCP1) [33], molecules that are highly expressed in tumor cells and tumor surrounding stromal cells [34]. Also, TAMs are recruited by other tumor-derived signals such as Macrophage Colony Stimulating Factor (M-CSF) or Granulocyte Macrophage Colony Stimulating Factor (GM-CSF), Vascular Endothelial Growth Factor (VEGF), CCL3/MIP1a, CCL4, CCL5, CCL8, angiopoeitin-2 and Platelet Derived Growth Factor (PDGF) [23,35]. Additionally, the overexpression of membrane bound 
Page 3 of 5

protein glypican-3 on HCC cellular membrane increases the recruitment of TAMs (Figure 1) [36].

TAMs subsequently undergo polarization depending on signals present in and around the tumor type. In HCC, the majority of the TAMs are of the M2 phenotype as this phenotype enables cancer proliferation and survival [24]. The tumor cells and tumor-derived cells therefore secrete certain factors, cytokines and chemokines that activate macrophage polarization towards the alternatively activated macrophage M2 state. Among the factors that recruit TAMs to the specific microenvironment, some equally possess a polarizing function. For instance, GM-CSF is a recruitment factor that equally polarizes monocytes towards the M1 macrophage phenotype in vitro, whereas M-CSF polarizes monocytes towards the M2 macrophage phenotype in vitro [37]. Other factors tend solely towards macrophage polarization. M1 macrophage polarization is induced by Toll Like Receptor (TLR) stimulation, bacterial infection and Interferon (IFN)- $\gamma$ stimulation. M2 macrophages are induced by various other signals, such as cytokines, Interleukin (IL)-4/13 and IL-10, glucocorticoids [32], anti-inflammatory molecules TGF- $\beta$, prostaglandin-E2 apoptotic cells and immune complexes (Figure 1)[29]. IFN- $\gamma$ orients macrophage polarization toward M1 through activation of signal transducer and activator of transcription (STAT) 1 pathway. In contrast, IL-4/13 and IL-10 activate the M2 phenotype through STAT6 and STAT3 respectively. SIFN- $\gamma[38]$. In order to counteract the proinflammatory M1 - Th1 immune response, cancer cells try to change the immune microenvironment by expressing factors that promote M2 macrophage polarization. HCC cells express high levels of IL-10, which, in turn, increases the expression and secretion of IL-4/13 (Figure 2) [34].

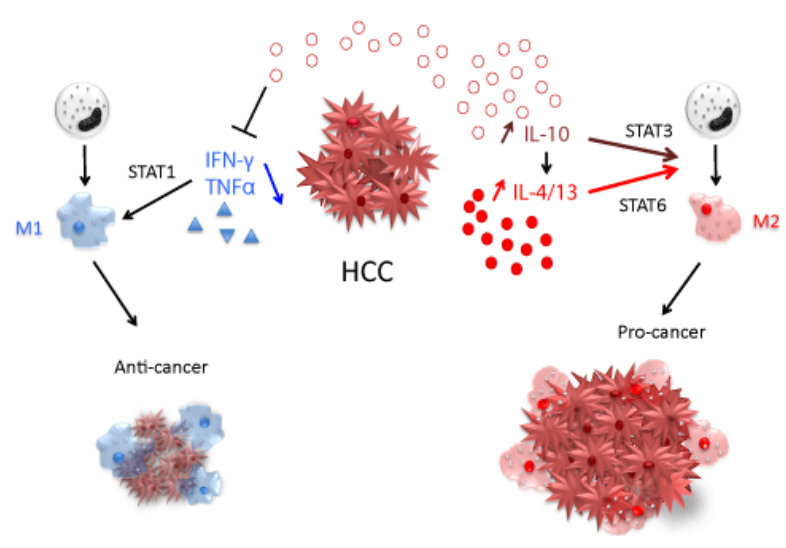

Figure 2: Plasticity of macrophages in tumor microenvironment and pathogenesis of HCC. Hepatocellular carcinoma cells secrete high levels of IL-10 (open circles), which induces increased production and secretion of IL-4 and IL-13 (closed circles). These interleukins increases M2 pro-cancer macrophage polarization, and inhibit the production and secretion of IFN $-\gamma$ and TNF (closed triangle), which tend towards an anti-tumor environment and M1 macrophage polarization.

These interleukins, as seen above, favor M2 macrophage polarization. The balance between activation of STAT3/STAT6 versus STAT1, which are controlled by IFN- $\gamma$ and interleukin concentrations, finely regulate macrophage expression and polarization [38]. The over-expression of the three interleukins (IL-4/13 and IL-10) activates
M2 polarization leading to Th2 immune response and inhibits the cytotoxic Th1 immune response. Particularly, IL-10 has an important role in M2 polarization; not only does it activate more M2 promoting cytokines (IL-4 and IL-13), but also it inhibits certain Th1 and cytotoxic promoting factors such as IFN $\gamma$ and IFN- $\alpha$ [39] and TNF- $\alpha$ (Figure 2)[40]. Such events occur as a consequence of cancer progression, immuno-bypassing and result in poor prognostic values.

The phenotypes of M1 and M2 macrophages can be characterized by their gene expression and transcriptional activities. M1 macrophages activate efficient immune effector cells with cytotoxic activities through $\mathrm{T}$ Helper 1 immune response. They are capable of eradicating tumor and microorganism cells, presenting high amounts of antigen and produce high amounts of T-cell stimulatory cytokines [30]. M2 macrophages, on the other hand, have a contrasting phenotype. Their phenotype can be characterized by the poor capability to present antigens, therefore inhibiting $\mathrm{T}$ cell recognition along with immunity against the pathological HCC cells. Also, the alternatively activated profile can be identified by the low expression of differentiation-associated macrophage antigens, such as carboxypeptidase M and CD51, but also tumor necrosis factor (TNF) and IL-12 [24]. Macrophages carrying low expressions of these factors favor tumor progression and proliferation as TNF promotes cancer regression and IL-12 promotes a cytotoxic, pro-inflammatory $\mathrm{T}$ Helper 1 immune response. In addition, M2 cells overexpress certain pro-survival factors such as Arginase I [41]. High levels of the enzyme Arginase I act as an adaptor mechanism to avoid the buildup of toxic nitric oxide (NO) in the tumor environment. It does so by bypassing NO production and producing polyamine and citrulline instead [41]. In addition, M2 macrophages express high levels of IL-10 and TGF- $\beta$ in order to maintain the M2 polarization state [23]. Finally, M2 macrophages express chemokines CCL17, CCL22 and CCL24 to promote $\mathrm{T}$ Helper 2 immune response, favoring angiogenesis, tissue remodeling and tissue repair [32].

\section{Future directions on targeting TAMs in HCC}

Recent studies demonstrate the importance of TAMs in many cancer types, showing that they account for multiple functions in tumor progression, metastasis, angiogenesis and immune evasion. Understanding the immunological microenvironment of hepatocellular carcinoma can provide valuable prognostic impact and also help plan for future immunity-based cancer treatments. TAMs are deeply involved in cancer survival and proliferation. Thus, targeting these macrophages [42] can prove to be an important therapeutic strategy for HCC. Most drugs, up to now, aim to block signaling pathways to inhibit pro-tumor factors in stromal cells. Increasing our knowledge on how macrophage polarization occurs in HCC and understanding the predominance of M2 macrophage polarization can help us to engineer drugs that counter-act the pro-cancer signals of tumor and tumor-associated stromal cells. Different signaling pathways can be targeted in order to produce an effective cocktail of anti-cancer drugs that boost the natural M1 macrophage immune response.

More concrete immunotherapeutic interventions targeting these cells may see the light in the near future as indicated by the very recent and fast track FDA-approved pembrolizumab (Keytruda) from Merck for the treatment of melanoma. This drug inhibits the interaction between programmed cell death receptor-1 (PD-1) and its ligands PDL1 and PD-L2. Since tumor cells express these immunosuppressive ligands, inhibition of the interaction between PD-1 and PD-L1 
increases the ability of the body's immune system to fight melanoma. Drugs targeting PD-1 pathway [43] such as monoclonal antibodies pembrolizumab and nivolumab may overcome immune resistance in HCC because of their immunostimulating activities on both $\mathrm{T}$ cells and macrophages.

Moreover, TAM infiltration should be evaluated by immunohistochemistry in tumor mass from biopsies as a part of a global in situ immune cell infiltrate in HCC or an "immunoscore" [44]. If validated in routine clinical settings, classifying the TAM density could serve to predict the recurrence of the tumor, equally providing prognostic information and guidance into selecting a specific immunotherapeutic drug for each patient. Finally, adjuvant immunotherapy targeting TAMs and other immune cells in remaining tumor masses following surgical intervention could also provide new horizons for immunotherapy and improve the outcome of patients in the future.

\section{References}

1. Llovet JM, Burroughs A, Bruix J (2003) Hepatocellular carcinoma. Lancet 362: 1907-1917.

2. Jemal A, Bray F, Center MM, Ferlay J, Ward E, et al. (2011) Global cancer statistics. CA Cancer J Clin 61: 69-90.

3. Di Bisceglie AM (2009) Hepatitis B and hepatocellular carcinoma. Hepatology 49: S56-60.

4. de Oliveria Andrade LJ, D'Oliveira A, Melo RC, De Souza EC, Costa Silva CA, et al. (2009) Association between hepatitis C and hepatocellular carcinoma. J Glob Infect Dis 1: 33-37.

5. Morgan TR, Mandayam S, Jamal MM (2004) Alcohol and hepatocellular carcinoma. Gastroenterology 127: S87-96.

6. Jones DE, Metcalf JV, Collier JD, Bassendine MF, James OF (1997) Hepatocellular carcinoma in primary biliary cirrhosis and its impact on outcomes. Hepatology 26: 1138-1142.

7. Caldwell SH, Crespo DM, Kang HS, Al-Osaimi AM (2004) Obesity and hepatocellular carcinoma. Gastroenterology 127: S97-103.

8. Kowdley KV (2004) Iron, hemochromatosis, and hepatocellular carcinoma. Gastroenterology 127: S79-86.

9. Hanahan D, Weinberg RA (2000) The hallmarks of cancer. Cell 100: 57-70.

10. Dvorak HF (1986) Tumors: wounds that do not heal. Similarities between tumor stroma generation and wound healing. N Engl J Med 315: 1650-1659.

11. Adam JK, Odhav B, Bhoola KD (2003) Immune responses in cancer. Pharmacol Ther 99: 113-132.

12. Pagès F, Galon J, Dieu-Nosjean MC, Tartour E, Sautès-Fridman C, et al. (2010) Immune infiltration in human tumors: a prognostic factor that should not be ignored. Oncogene 29: 1093-1102.

13. Hanahan D, Weinberg RA (2011) Hallmarks of cancer: the next generation. Cell 144: 646-674.

14. Grivennikov SI, Greten FR, Karin M (2010) Immunity, inflammation, and cancer. Cell 140: 883-899.

15. Qian BZ, Pollard JW (2010) Macrophage diversity enhances tumor progression and metastasis. Cell 141:39-51.

16. Vajdic CM, van Leeuwen MT (2009) Cancer incidence and risk factors after solid organ transplantation. Int J Cancer 125: 1747-1754.

17. Kim R, Emi M, Tanabe K (2007) Cancer immunoediting from immune surveillance to immune escape. Immunology 121: 1-14.

18. Yang JD, Nakamura I, Roberts LR (2011) The tumor microenvironment in hepatocellular carcinoma: current status and therapeutic targets. Semin Cancer Biol 21: 35-43.

19. Chew V, Chen J, Lee D, Loh E, Lee J, et al. (2012) Chemokine-driven lymphocyte infiltration: an early intratumoural event determining longterm survival in resectable hepatocellular carcinoma. Gut 61: 427-438.
20. Fu J, Xu D, Liu Z, Shi M, Zhao P, et al. (2007) Increased regulatory T cells correlate with CD8 T-cell impairment and poor survival in hepatocellular carcinoma patients. Gastroenterology 132: 2328-2339.

21. Pollard JW (2004) Tumour-educated macrophages promote tumour progression and metastasis. Nat Rev Cancer 4: 71-78.

22. Siveen KS, Kuttan G (2009) Role of macrophages in tumour progression. Immunol Lett 123: 97-102.

23. Capece D, Fischietti M, Verzella D, Gaggiano A, Cicciarelli G, et al. (2013) The inflammatory microenvironment in hepatocellular carcinoma: a pivotal role for tumor-associated macrophages. Biomed Res Int 2013: 187204.

24. Shirabe K, Mano Y, Muto J, Matono R, Motomura T, et al. (2012) Role of tumor-associated macrophages in the progression of hepatocellular carcinoma. Surg Today 42: 1-7.

25. Liaskou E, Zimmermann HW, Li KK, Oo YH, Suresh S, et al. (2013) Monocyte subsets in human liver disease show distinct phenotypic and functional characteristics. Hepatology 57: 385-398.

26. Budhu A, Forgues M, Ye QH, Jia HL, He P, et al. (2006) Prediction of venous metastases, recurrence, and prognosis in hepatocellular carcinoma based on a unique immune response signature of the liver microenvironment. Cancer Cell 10: 99-111.

27. Porta C, Larghi P, Rimoldi M, Totaro MG, Allavena P, et al. (2009) Cellular and molecular pathways linking inflammation and cancer. Immunobiology 214: 761-777.

28. Martinez FO, Sica A, Mantovani A, Locati M (2008) Macrophage activation and polarization. Front Biosci 13: 453-461.

29. Sica A, Mantovani A (2012) Macrophage plasticity and polarization: in vivo veritas. J Clin Invest 122: 787-795.

30. Mantovani A, Sozzani S, Locati M, Allavena P, Sica A (2002) Macrophage polarization: tumor-associated macrophages as a paradigm for polarized M2 mononuclear phagocytes. Trends Immunol 23: 549-555.

31. Hao NB, Lü MH, Fan YH, Cao YL, Zhang ZR, et al. (2012) Macrophages in tumor microenvironments and the progression of tumors. Clin Dev Immunol 2012: 948098.

32. Solinas G, Germano G, Mantovani A, Allavena P (2009) Tumorassociated macrophages (TAM) as major players of the cancer-related inflammation. J Leukoc Biol 86: 1065-1073.

33. Coussens LM, Werb Z (2002) Inflammation and cancer. Nature 420: 860-867.

34. Mantovani A, Germano G, Marchesi F, Locatelli M, Biswas SK (2011) Cancer-promoting tumor-associated macrophages: new vistas and open questions. Eur J Immunol 41: 2522-2525.

35. Murdoch C, Giannoudis A, Lewis CE (2004) Mechanisms regulating the recruitment of macrophages into hypoxic areas of tumors and other ischemic tissues. Blood 104: 2224-2234.

36. Takai H, Ashihara M, Ishiguro T, Terashima H, Watanabe $\mathrm{T}$, et al. (2009) Involvement of glypican-3 in the recruitment of M2-polarized tumorassociated macrophages in hepatocellular carcinoma. Cancer Biol Ther 8: 2329-2338.

37. Rey-Giraud F, Hafner M, Ries CH (2012) In vitro generation of monocyte-derived macrophages under serum-free conditions improves their tumor promoting functions. PLoS One 7: e42656.

38. Sica A, Invernizzi P, Mantovani A (2014) Macrophage plasticity and polarization in liver homeostasis and pathology. Hepatology 59: 2034-2042.

39. Ito S, Ansari P, Sakatsume M, Dickensheets H, Vazquez N, et al. (1999) Interleukin-10 inhibits expression of both interferon alpha- and interferon gamma- induced genes by suppressing tyrosine phosphorylation of STAT1. Blood 93: 1456-1463.

40. Shin DI, Banning U, Kim YM, Verheyen J, Hannen M, et al. (1999) Interleukin 10 inhibits TNF-alpha production in human monocytes independently of interleukin 12 and interleukin 1 beta. Immunol Invest 28: 165-175.

41. Gabrilovich DI, Nagaraj S (2009) Myeloid-derived suppressor cells as regulators of the immune system. Nat Rev Immunol 9: 162-174. 
Citation: Lewis M, Merched AJ (2014) Tumor-Associated Macrophages, Inflammation and Pathogenesis of Hepatocellular Carcinoma. J Mol Genet Med 8: 132. doi:10.4172/1747-0862.1000132

Page 5 of 5

42. Germano G, Frapolli R, Belgiovine C, Anselmo A, Pesce S, et al. (2013) Role of macrophage targeting in the antitumor activity of trabectedin. Cancer Cell 23: 249-262.

43. Mamalis A, Garcha M, Jagdeo J (2014) Targeting the PD-1 pathway: a promising future for the treatment of melanoma. Arch Dermatol Res 306: 511-519.
44. Galon J, Mlecnik B, Bindea G, Angell HK, Berger A, et al. (2014) Towards the introduction of the 'Immunoscore' in the classification of malignant tumours. J Pathol 232: 199-209. 